\title{
SUBSTITUIÇÃO DOS PARÂMETROS DO MODELO DE GREEN- AMPT-MEIN-LARSON PARA ESTIMATIVA DA INFILTRAÇÃO EM ALGUNS SOLOS DO BRASIL ${ }^{(1)}$
}

\author{
Roberto Avelino Cecílio ${ }^{(2)}$, Mauro Aparecido Martinez ${ }^{(3)}$, Fernando \\ Falco Pruski ${ }^{(3)}$, Demetrius David da Silva ${ }^{(4)} \&$ Wendy Fonseca \\ Ataíde ${ }^{(5)}$
}

\begin{abstract}
RESUMO
A partir de uma análise detalhada do processo de infiltração de água no solo, foram propostas alternativas para adequação dos parâmetros de entrada do modelo de Green-Ampt-Mein-Larson (GAML), na tentativa de melhorar a eficiência da estimativa da infiltração em alguns solos brasileiros. As adequações consistiram em substituir a condutividade hidráulica do solo saturado $\left(K_{0}\right)$ pela taxa de infiltração estável (Tie) e o teor de água do solo saturado $\left(\theta_{\mathrm{S}}\right)$ pelo teor de água na zona de transmissão $\left(\theta_{\mathrm{w}}\right)$, além de estabelecer uma equação para estimativa do potencial matricial na frente de umedecimento $\left(\psi_{\mathrm{f}}\right)$ com base nos parâmetros do modelo de curva de retenção de água de Brooks \& Corey. Avaliou-se o desempenho do conjunto de adequações propostas (GAML-t) para estimativa da infiltração de água em três solos: Latossolo Vermelho-Amarelo, Latossolo Vermelho e Argissolo Vermelho. O desempenho do GAML-t foi comparado com o obtido pelo GAML aplicado em sua forma original e adequado segundo cinco diferentes formas, evidenciando-se que o primeiro obteve melhor desempenho nos três solos estudados.
\end{abstract}

Termos de indexação: modelagem hidrológica, potencial matricial, condutividade hidráulica.

\footnotetext{
(1) Parte da Tese de Doutorado apresentada pelo primeiro autor à UFV em 2005. Recebido para publicação em setembro de 2006 e aprovado em maio de 2007.

${ }^{(2)}$ Professor Adjunto do Departamento de Engenharia Florestal, Universidade Federal do Espírito Santo - UFES. Caixa Postal 16, CEP 29500-000 Alegre (ES). E-mail: racecilio@yahoo.com.br

${ }^{(3)}$ Professor do Departamento de Engenharia Agrícola, Universidade Federal de Viçosa - UFV. CEP 36570-000 Viçosa (MG). E-mail:mmauro@fv.br; ffpruski@ufv.br

(4) Engenheiro-Agrícola e Ambiental, Doutorando em Tecnologia Ambiental e Recursos Hídricos. Faculdade de Tecnologia, Universidade de Brasília - UnB. CEP70910-900. Brasília (DF). E-mail: w_ataide@uol.com.br

${ }^{(5)}$ Doutorando em Tecnologia Ambiental e Recursos Hídricos. Faculdade de Tecnologia, UnB. E-mail: david@ufv.br
} 


\title{
SUMMARY: ADJUSTMENTOFGREEN-AMPT-MEIN-LARSONPARAMETERS TO PREDICT INFILTRATION IN SOME BRAZILIAN SOILS
}

\begin{abstract}
Based on a detailed analysis of the process of water infiltration into the soil, an alternative is proposed to adjust the input parameters of the Green-Ampt-Mein-Larson (GAML) model to optimize infiltration estimates of some Brazilian soils. The adjustments consisted of the replacement of the parameters saturated hydraulic conductivity $\left(K_{0}\right)$ by stable infiltration rate (Tie) and soil moisture saturation $\left(\theta_{s}\right)$ by water content in the transmission zone $\left(\theta_{w}\right)$. Moreover, an equation was established to estimate the matric potencial at the wetting front $\left(y_{f}\right)$, based on the parameters of the water retention curve of Brooks and Corey. The performance of the set of proposed adjustments (GAML-t) was evaluated for the estimation of water infiltration in three soil types. The performance of $G A M L-t$ was compared with the original GAML and adjusted to five different situations. GAML-t performed best in the three soils under study.
\end{abstract}

Index terms: hydrologic modeling, soil water matric potential, hydraulic conductivity.

\section{INTRODUÇÃO}

Infiltração é o processo de passagem da água da superfície para o interior do solo. Sabe-se que a melhoria das condições de infiltração da água reduz a intensidade do processo erosivo e estimula a recarga dos aqüíferos subterrâneos, o aumento do escoamento de base e a redução das vazões máximas dos cursos d'água, além de ser determinante do balanço hídrico na região da zona radicular das culturas. Dessa forma, verifica-se que o conhecimento desse processo é fundamental para o gerenciamento racional dos recursos hídricos, o manejo e conservação do solo e da água, o dimensionamento de estruturas hidráulicas e a condução da agricultura irrigada (Brandão et al., 2006).

A demanda da sociedade por modelos que permitam melhor previsão hidrológica aumenta quando da notificação de inundações e dos prejuízos relacionados a estas (Palacios-Vélez et al., 1998). O desenvolvimento de modelos hidrológicos mais eficientes, entretanto, requer esforço contínuo de pesquisa da comunidade acadêmica, visto que esses modelos necessitam de informações consistentes sobre precipitação, infiltração, escoamento, topografia, solo, entre outras. Nesse contexto, destaca-se a necessidade de uma estimativa mais confiável do processo de infiltração para modelagem do movimento de água no solo, a determinação das perdas de solo e água decorrentes de processos erosivos e a previsão do potencial de contaminação de aqüíferos subterrâneos.

Dentre os modelos existentes para estimativa da infiltração de água no solo sob condições de precipitação, aquele apresentado por Mein \& Larson (1973), denominado de Green-Ampt-Mein-Larson (GAML), é um dos mais empregados devido à sua relativa simplicidade operacional. O GAML calcula a taxa de infiltração, a partir do momento em que ocorre o empoçamento de água sobre a superfície, por meio da equação 1:

$$
\mathrm{Ti}=\mathrm{K}_{0}\left(1+\frac{\psi_{\mathrm{f}}\left(\theta_{\mathrm{s}}-\theta_{\mathrm{i}}\right)}{\mathrm{I}}\right)
$$

em que Ti é taxa de infiltração $\left(\mathrm{mm} \mathrm{h}^{-1}\right) ; \mathrm{K}_{0}$, a condutividade hidráulica do solo saturado $\left(\mathrm{mm} \mathrm{h}^{-1}\right)$; $\psi_{\mathrm{f}}$, o potencial matricial na frente de umedecimento $(\mathrm{mm}) ; \theta_{\mathrm{s}}$, o teor de água do solo saturado $\left(\mathrm{m}^{3} \mathrm{~m}^{-3}\right) ; \theta_{\mathrm{i}}$, o teor de água inicial do solo $\left(\mathrm{m}^{3} \mathrm{~m}^{-3}\right)$; e I, a lâmina infiltrada $(\mathrm{mm})$.

A lâmina de água infiltrada no solo até a ocorrência do empoçamento de água sobre a superfície $\left(\mathrm{I}_{0}\right.$, em $\mathrm{mm}$ ) é calculada pela equação 2 , sendo o tempo necessário para tal ( $t_{p}$, em horas) calculado pela razão entre $\mathrm{I}_{0}$ e a intensidade de precipitação $\left(\mathrm{i}_{\mathrm{p}}, \mathrm{em} \mathrm{mm} \mathrm{h}^{-1}\right)$. Durante o tempo $t_{p}$, tem-se que $\mathrm{Ti}=\mathrm{i}_{\mathrm{p}}$.

$$
\mathrm{I}_{0}=\frac{\psi_{\mathrm{f}}}{\frac{\mathrm{i}_{\mathrm{p}}}{\mathrm{K}_{0}}-1}\left(\theta_{\mathrm{s}}-\theta_{\mathrm{i}}\right)
$$

A equação 1 pode ser explicitada no sentido de calcular a taxa de infiltração em função do tempo, ficando expressa na seguinte forma (Mein \& Larson, 1973):

$$
\mathrm{K}_{0}\left(\mathrm{t}-\mathrm{t}_{\mathrm{p}}+\mathrm{t}_{\mathrm{s}}\right)=\mathrm{I}-\psi_{\mathrm{f}}\left(\theta_{\mathrm{s}}-\theta_{\mathrm{i}}\right) \ln \left[1+\frac{\mathrm{I}}{\psi_{\mathrm{f}}\left(\theta_{\mathrm{s}}-\theta_{\mathrm{i}}\right)}\right]
$$

em que t é o tempo decorrido desde o início da infiltração (horas) e $t_{\mathrm{s}}$ (em horas) é o tempo necessário para infiltrar a lâmina $\mathrm{I}_{0}$ sob condições de saturação da camada superficial do solo, calculado pela equação 3 , substituindo-se o termo $\left(t-t_{p}+t_{s}\right)$ por $t_{s}$ e o termo I por $I_{o}$.

Podem-se destacar, entretanto, duas limitações para utilização do GAML: os parâmetros de entrada $\mathrm{K}_{0}, \psi_{\mathrm{f}}$ e $\theta_{\mathrm{s}}$ não representam efetivamente as condições reais de ocorrência da infiltração; e a dificuldade e pouca confiabilidade nos métodos de obtenção destes parâmetros (Cecílio et al., 2003). Propostas simples para contornar esses problemas vêm sendo feitas há décadas por diversos pesquisadores, que desenvolveram 
metodologias para adequação dos parâmetros de entrada do GAML. Contudo, muitas dessas propostas baseiam-se em equações empíricas, derivadas de dados experimentais, portanto, nem sempre aplicáveis em qualquer situação ou em qualquer tipo de solo.

Este trabalho teve como objetivo substituir parâmetros utilizados no modelo GAML para estimativa da infiltração de água em três diferentes solos brasileiros.

\section{MATERIAL E MÉTODOS}

\section{Proposta de adequação dos parâmetros de entrada do GAML}

O conjunto de adequações propostas para o modelo GAML, a partir de agora denominado GAML-t, consistiu em substituir três dos parâmetros de entrada do modelo: $\theta_{\mathrm{s}}, \mathrm{K}_{0}$ e $\psi_{\mathrm{f}}$.

Tomando por base outras adequações feitas por Brakensiek \& Onstad (1977), Slack (1980), Cecílio et al. (2003) e Mello (2003), propõe-se que o parâmetro $\theta_{\mathrm{s}}$ seja substituído pelo teor de água da zona de transmissão $\left(\theta_{\mathrm{w}}\right)$, que é, efetivamente, o teor de água que a maior parte do perfil de solo atinge durante a infiltração.

O teor de água ao longo da frente de umedecimento varia entre $\theta_{\mathrm{w}}$ e $\theta_{\mathrm{i}}$, portanto, considera-se que o cálculo de $\psi_{\mathrm{f}}$ deve ser feito a partir da integração da curva de condutividade hidráulica relativa $\left(\mathrm{K}_{\mathrm{r}}\right)$ em função do potencial matricial ( $\psi$ ) (equação 3), conforme proposta feita por Mein \& Larson (1973), modificada por Slack (1980).

$$
\psi_{\mathrm{f}}=\int_{\frac{\mathrm{K}\left(\theta_{\mathrm{i}}\right)}{\mathrm{K}_{0}}}^{\frac{\mathrm{Kw}}{\mathrm{K}_{0}}} \frac{\psi d \mathrm{dK}_{\mathrm{r}}}{\left(\frac{\mathrm{KW}}{\mathrm{K}_{0}}-\frac{\mathrm{K}\left(\theta_{\mathrm{i}}\right)}{\mathrm{K}_{0}}\right)}
$$

em que Kw é a condutividade hidráulica do solo na umidade $\theta_{\mathrm{w}}$ e Kr é a condutividade hidráulica relativa (adimensional), igual a $\mathrm{K}(\theta) / \mathrm{K}_{0}$.

Desenvolvendo a equação 4 com utilização da equação 5 (Alexander \& Skaggs, 1986) para expressar a condutividade hidráulica relativa, obtém-se a equação 6 , adotada para o cálculo de $\psi_{\text {f }}$.

$$
\mathrm{K}_{\mathrm{r}}=\frac{\mathrm{K}(\theta(\psi))}{\mathrm{K}_{0}}=\left[\frac{\psi_{\mathrm{b}}}{\psi(\theta)}\right]^{2+3 \lambda}
$$

em que $\psi_{b}$ é o potencial matricial de entrada de ar $(\mathrm{mm})$ e $\lambda$ é o índice de distribuição de tamanho de poros (adimensional), obtidos por intermédio do ajuste do modelo de curva de retenção de água no solo proposto por Brooks \& Corey (1964).

$$
\psi_{\mathrm{f}}=\frac{\psi_{\mathrm{b}}(2+3 \lambda)\left[\operatorname{Kr}\left(\theta_{\mathrm{w}}\right)^{\frac{3 \lambda+1}{3 \lambda+2}}-\operatorname{Kr}\left(\theta_{\mathrm{i}}\right)^{\frac{3 \lambda+1}{3 \lambda+2}}\right]}{\left[\operatorname{Kr}\left(\theta_{\mathrm{w}}\right)-\operatorname{Kr}\left(\theta_{\mathrm{i}}\right)\right][3 \lambda+1]}
$$

Uma das inconveniências do modelo refere-se à obtenção confiável de $\mathrm{K}_{0}$, que leva a coeficientes de variação superiores a 70 \% (Mesquita \& Moraes, 2004). Esse problema pode ser minimizado utilizando-se a taxa de infiltração estável (Tie) como representativa da condutividade hidráulica de todo o perfil de solo, conforme também proposto por Silva \& Kato (1998).

\section{Testes experimentais}

Os testes experimentais consistiram na determinação da lâmina infiltrada e da taxa de infiltração da água, sob chuva simulada, em perfis de solo acondicionados em colunas de PVC. Para isso, foram utilizados materiais de solo pertencentes ao horizonte A de três classes distintas: Latossolo Vermelho-Amarelo (LVA), Argissolo Vermelho (PV) e Latossolo Vermelho (LV), realizando-se três testes experimentais para cada classe. A análise textural dos solos é apresentada no quadro 1.

Utilizaram-se três colunas de PVC com 0,20 m de diâmetro e altura útil de 0,48 m (perfis dos solos PV e $\mathrm{LV}$ ) ou de 0,78 m (perfis do LVA). Cada coluna foi montada sobre tampão de PVC de $20 \mathrm{~cm}$ de diâmetro, sendo seu fundo aberto com um orifício de $1 \mathrm{~cm}$ de diâmetro e seu interior preenchido com brita e areia grossa, para permitir a rápida drenagem da água. A fim de facilitar o adensamento dos materiais de solo dentro das colunas e o posterior desmanche destas, elas foram construídas com módulos de PVC de $10 \mathrm{~cm}$ de altura, unidos por intermédio de cola de silicone e tira de borracha para vedação. O módulo de PVC localizado na parte superior das colunas foi dotado de uma calha para condução do escoamento superficial. A base desta calha localizava-se a $2 \mathrm{~cm}$ do topo da coluna de solo, fazendo com que a altura útil deste último módulo fosse de apenas $8 \mathrm{~cm}$. A fim de minimizar a ocorrência de caminhos preferenciais para água entre as paredes internas das colunas e o material de solo, procedeu-se à colagem de areia grossa nas paredes destas, aumentando assim a sua rugosidade.

Antes de se iniciar o preenchimento das colunas com os materiais de solo, estes foram umedecidos e guardados em recipientes vedados durante uma semana, com o intuito de homogeneizar a umidade de todo o material. Posteriormente, a umidade do material de solo contido nos recipientes foi determinada, com o objetivo de calcular a massa de solo úmido necessária para adensar camadas de 4 e $5 \mathrm{~cm}$ de solo dentro das colunas de PVC. A massa de solo utilizada para adensamento de cada camada foi calculada de modo que a massa específica do solo adensado dentro da coluna se aproximasse da massa específica observada em campo. As camadas foram adensadas individualmente a partir de carga exercida com a queda de um peso em uma placa de PVC com diâmetro de $20 \mathrm{~cm}$, colocada sobre o solo.

A condução dos testes experimentais consistiu, essencialmente, em aplicar chuva simulada com 
Quadro 1. Análise textural dos solos utilizados no experimento

\begin{tabular}{lrrrrll}
\hline Solo & Areia grossa & Areia fina & Silte & Argila & Classificação textural & Estrutura \\
\hline & & & & & \\
LVA & 13 & 10 & 7 & 70 & muito-argilosa & forte pequena / granular \\
PV & 7 & 9 & 25 & 59 & Argila & forte média / blocos subangulares \\
LV & 26 & 52 & 2 & 20 & franco-arenosa & forte pequena/média / granular \\
\hline
\end{tabular}

intensidade constante sobre cada perfil de solo contido nas colunas de PVC e coletar todo o escoamento superficial produzido. Antes de se iniciar a aplicação de água sobre o perfil de solo, sua superfície era coberta com manta de tecido sintético altamente permeável (bidim), a fim de minimizar os efeitos do selamento superficial.

Para aplicação da chuva simulada foi utilizado um simulador estacionário de bicos múltiplos e oscilantes construído na UFV, conforme modelo proposto pelo National Soil Erosion Research Laboratory, vinculado ao Departamento de Agricultura dos Estados Unidos. O simulador apresentava bocais do tipo Veejet 80.100, espaçados de $1,10 \mathrm{~m}$, operando com pressão de serviço de $34,5 \mathrm{kPa}$. As intensidades de precipitação $\left(i_{\mathrm{p}}\right)$ aplicadas sobre os perfis de solo foram de $530,558 \mathrm{e}$ $574 \mathrm{~mm} \mathrm{~h}^{-1}$ nos três testes experimentais realizados no LV; 486, 442 e $398 \mathrm{~mm} \mathrm{~h}^{-1}$ naqueles feitos com o LVA; e 211, 225 e $274 \mathrm{~mm} \mathrm{~h}^{-1}$ nos realizados com o PV.

Sondas de TDR foram inseridas paralelas à superfície do solo nas profundidades de $6,11,16,21$, $26,31,36,41$ e $46 \mathrm{~cm}$ nos testes realizados com os solos PV e LV; e nas profundidades de 6, 16, 26, 36, $41,46,51,56,61,66,71$ e $76 \mathrm{~cm}$ nos testes realizados com o LVA. Essas sondas foram utilizadas para medição do teor de água tanto inicial como final do solo.

O escoamento superficial ocorrido durante a aplicação de chuva simulada foi conduzido a uma estrutura de coleta por intermédio da calha instalada na parte superior da coluna de PVC. A estrutura de coleta era composta por dois reservatórios, que se comunicavam por intermédio de uma mangueira flexível. O primeiro reservatório destinava-se a coletar toda a água escoada, enquanto no segundo foi instalado um equipamento medidor de nível de água, denominado Thalimedes, que registrava e armazenava a altura da lâmina de água dentro da estrutura de coleta.

$\mathrm{O}$ volume de água infiltrada em um intervalo de tempo $\Delta \mathrm{t}\left(\mathrm{V}_{\mathrm{inf}}\right)$ foi obtido indiretamente por meio da diferença entre os volumes precipitado $\left(V_{\text {prec }}\right)$ e escoado $\left(\mathrm{V}_{\mathrm{esc}}\right)$. A taxa de infiltração foi determinada dividindose a lâmina infiltrada pelo intervalo de tempo entre duas leituras sucessivas.
Os testes foram encerrados no momento em que ocorreu a saída de água pelo dreno localizado na parte inferior da coluna de PVC. Considerou-se que a taxa de infiltração estável da água no solo (Tie) foi atingida após se verificar que a taxa de variação do nível d'água dentro da estrutura de coleta tornou-se constante. Os valores da Tie para os nove testes experimentais são apresentados no quadro 2.

Os teores de água da zona de transmissão, apresentados no quadro 2 , foram calculados fazendose a média dos teores de água determinados, por intermédio do TDR, ao final dos testes experimentais. Para isso, foram utilizados apenas os teores de água medidos nas cinco sondas localizadas na parte inferior das colunas de solo, uma vez que se percebia que o teor de água medido nas sondas superiores era praticamente o mesmo do solo saturado, indicando que o solo atingia a saturação nas camadas superiores (até cerca de $25 \mathrm{~cm})$.

Posteriormente, amostras de solo foram retiradas para determinação da porosidade, da densidade do solo e também da condutividade hidráulica do solo saturado, pelo método do permeâmetro de carga constante. Também foram retiradas amostras para determinação da curva de retenção e posterior ajuste dos parâmetros $\lambda$ e $\psi_{\mathrm{b}}$ do modelo de Brooks \& Corey (1964). O quadro 2 apresenta os valores relativos às características determinadas para cada solo.

\section{Simulações}

Foram realizadas simulações do processo de infiltração utilizando-se o modelo GAML e o GAML-t, tanto em sua forma original quanto modificado a partir de adequações dos parâmetros de entrada recomendadas por Cecílio et al. (2003) e por Silva \& Kato (1998). No quadro 3 são apresentadas as simulações feitas para cada teste experimental, com os respectivos parâmetros de entrada usados.

O desempenho das simulações foi avaliado utilizando-se o índice de confiança ajustado (c'), adaptado do índice de confiança (c), proposto por Camargo \& Sentelhas (1997). O índice c' é calculado pelo produto entre o coeficiente de correlação $(r)$ e o índice de concordância ajustado (d'), variando entre zero (nenhuma concordância) e 1 (concordância 
Quadro 2. Atributos físicos dos solos utilizados

\begin{tabular}{|c|c|c|c|c|c|c|c|c|c|}
\hline Solo & Teste & $\theta_{\mathrm{i}}$ & $\theta_{\mathrm{s}}$ & $\theta_{\mathrm{w}}$ & da & $\mathbf{K}_{0}$ & Tie & $\psi_{\mathbf{b}}$ & $\lambda$ \\
\hline \multirow{4}{*}{ LVA } & & 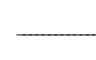 & $\mathrm{m}^{3} \mathrm{~m}^{-3}$ & + & $\mathrm{kg} \mathrm{dm^{-3 }}$ & \multicolumn{2}{|c|}{$\mathrm{mm} \mathrm{h}^{-1}$} & \multicolumn{2}{|l|}{$\mathrm{cm}$} \\
\hline & 1 & 0,327 & 0,541 & 0,521 & 1,05 & 112,1 & 130,3 & 8,7 & 0,4032 \\
\hline & 2 & 0,320 & 0,537 & 0,509 & 1,06 & 129,5 & 72,5 & 9,1 & 0,4032 \\
\hline & 3 & 0,318 & 0,543 & 0,524 & 1,07 & 106,0 & 107,6 & 9,1 & 0,4032 \\
\hline \multirow[t]{3}{*}{ PV } & 1 & 0,300 & 0,543 & 0,300 & 1,19 & 35,5 & 78,9 & 10,4 & 0,3572 \\
\hline & 2 & 0,297 & 0,542 & 0,297 & 1,19 & 50,7 & 132,4 & 10,6 & 0,3572 \\
\hline & 3 & 0,289 & 0,541 & 0,289 & 1,19 & 83,2 & 195,7 & 10,7 & 0,3572 \\
\hline \multirow[t]{3}{*}{ LV } & 1 & 0,113 & 0,479 & 0,398 & 1,42 & 102,6 & 253,1 & 13,2 & 0,7470 \\
\hline & 2 & 0,100 & 0,477 & 0,413 & 1,43 & 99,1 & 253,3 & 13,2 & 0,7470 \\
\hline & 3 & 0,093 & 0,474 & 0,424 & 1,44 & 101,6 & 227,4 & 13,6 & 0,7470 \\
\hline
\end{tabular}

$\theta_{i}$ : teor de água inicial do solo; $\theta_{\mathrm{s}}$ : teor de água do solo saturado (igual à porosidade do solo); $\theta_{\mathrm{w}}$ : teor de água da zona de transmissão; da: massa específica do solo; $\mathrm{K}_{0}$ : condutividade hidráulica do solo saturado; Tie: taxa de infiltração estável; $\psi_{\mathrm{b}}$ : potencial matricial de entrada de ar; $\lambda$ : índice de distribuição de tamanho de poros.

Quadro 3. Parâmetros modificados na realização das simulações

\begin{tabular}{|c|c|c|c|c|}
\hline Simulação & $\theta_{\mathrm{s}}$ & $\mathbf{K}_{0}$ & $\psi_{\mathrm{f}}$ & Fonte \\
\hline $\mathrm{S} 1$ & $\theta_{\mathrm{w}}$ & Tie & eq. 6 & GAML-t \\
\hline $\mathrm{S} 2$ & $\theta_{\mathrm{s}}$ & $\mathrm{K}_{0}$ & $\psi_{\mathrm{f}}=\int_{0,01}^{1} \psi \mathrm{dK_{r }}$ & Mein \& Larson (1973) \\
\hline S3 & $\theta_{\mathrm{w}}$ & $0,5 \mathrm{~K}_{0}$ & $\psi(\theta \mathrm{i})$ & Cecílio et al. (2003) \\
\hline $\mathrm{S} 4$ & $\theta_{\mathrm{w}}$ & $\mathrm{K}_{0}$ & $\psi_{\mathrm{f}}=0,01 \mathrm{e}^{\alpha}$ & Cecílio et al. (2003) \\
\hline S5 & $\theta_{\mathrm{w}}$ & Tie & $\psi_{\mathrm{f}}=0,01 \mathrm{e}^{\alpha}$ & Cecílio et al. (2003) \\
\hline $\mathrm{S} 6$ & $\theta_{\mathrm{w}}$ & Tie & {$[\psi(\theta \mathrm{i})+\psi(\theta \mathrm{w})] / 2$} & Cecílio et al. (2003) \\
\hline $\mathrm{S} 7$ & $\theta_{\mathrm{s}}$ & Tie & $\psi_{\mathrm{f}}=\int_{0,01}^{1} \psi \mathrm{dK} \mathrm{r}_{\mathrm{r}}$ & Silva \& Kato (1998) \\
\hline
\end{tabular}

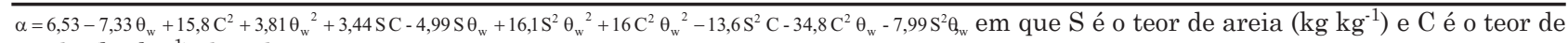
argila $\left(\mathrm{kg} \mathrm{kg}^{-1}\right)$ do solo.

perfeita). O índice d', proposto por Legates \& McCabe Jr. (1999), é adaptado do índice de concordância de Willmontt (1981), sendo calculado pela equação:

$$
d^{\prime}=1-\frac{\sum_{i=1}^{J}\left|O_{i}-E_{i}\right|}{\sum_{i=1}^{J}\left(\left|E_{i}-\bar{O}\right|+\left|O_{i}-\bar{O}\right|\right)}
$$

em que J é o número de observações; Oi, o valor observado experimentalmente; Ei, o valor estimado pelo modelo; e $\overline{0}$, a média dos valores observados experimentalmente.

No quadro 4 são apresentados os critérios de avaliação do desempenho de modelos quanto ao seu índice de confiança. 
Quadro 4. Análise do desempenho com base no índice de confiança ajustado (c') (adaptado de Camargo \& Sentelhas, 1997)

\begin{tabular}{cl}
\hline Valor de c' & Desempenho \\
\hline & \\
0,85 & Ótimo \\
0,76 a 0,85 & Muito bom \\
0,66 a 0,75 & Bom \\
0,61 a 0,65 & Mediano \\
0,51 a 0,60 & Sofrível \\
0,41 a 0,50 & Mau \\
$\leq 0,40$ & Péssimo \\
\hline
\end{tabular}

\section{RESULTADOS E DISCUSSÃO}

As curvas simuladas de Ti e I ao longo do tempo estão indicadas nas figuras 1, 2 e 3, bem como os seus valores experimentais nos testes realizados nos perfis dos solos LVA, PV e LV, respectivamente. No quadro 5 são apresentados o índice c' e o desempenho das simulações realizadas na estimativa de I e Ti feita nos nove testes experimentais.

Verifica-se que nos testes experimentais relativos ao LVA as simulações S3, S4, S5 e S6 (adequações apresentadas por Cecílio et al., 2003) não foram capazes de estimar adequadamente o processo de infiltração de água no solo, apresentando superestimativas de Ti e I (Figura 1 e Quadro 5). Interessante ressaltar que o LVA foi o mesmo solo utilizado por Cecílio et al. (2003) para propor as adequações dos parâmetros de GAML. Todavia, os referidos autores trabalharam com solo em condições de estratificação, estimando a infiltração nos horizontes A, B e C, e não apenas no material de solo do horizonte A, como no presente trabalho. Os autores ainda recomendaram a aplicação dessas formas de adequação para alta umidade inicial do solo, condição diferente da aplicada no trabalho em apreço. Nota-se também que os resultados das simulações S2 (Mein \& Larson, 1973), S1 (GAML-t) e S7 (parâmetros modificados por Silva \& Kato, 1998) são muito próximos, exceção feita à simulação $\mathrm{S} 2$ no segundo (a)
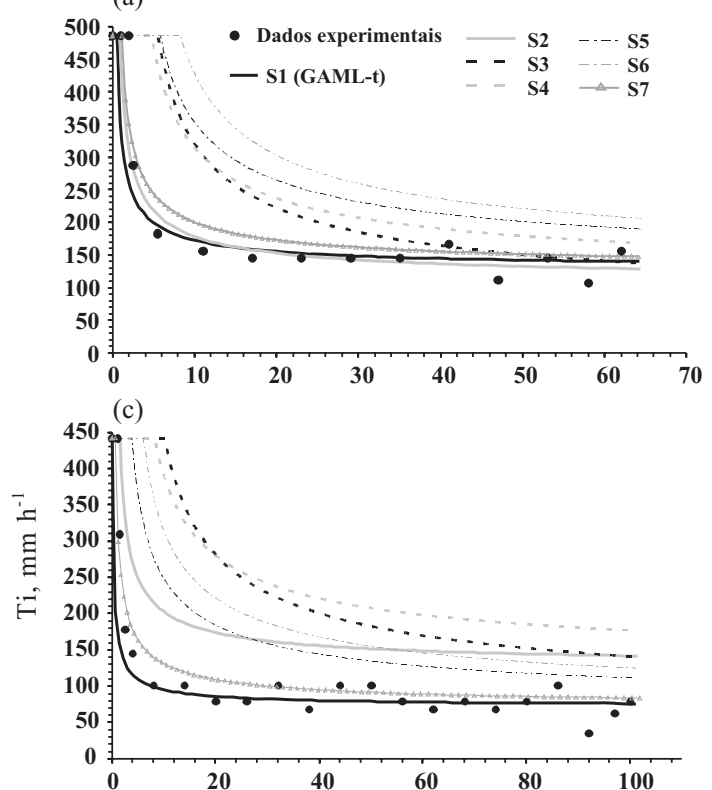

(e)

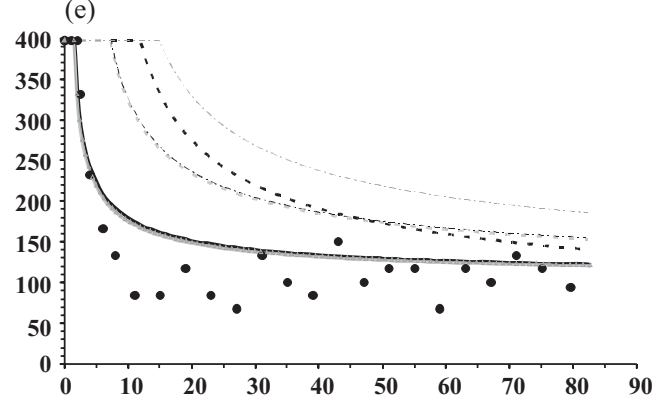

(b)

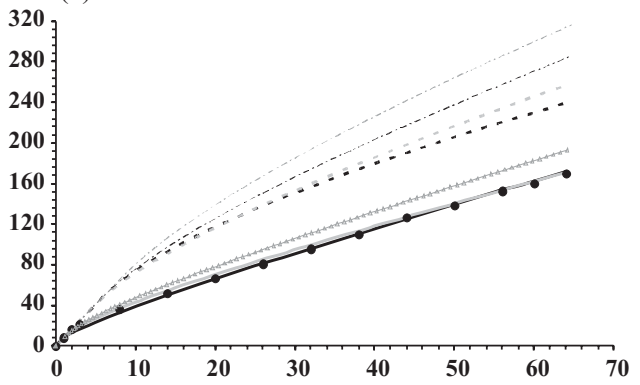

(d)

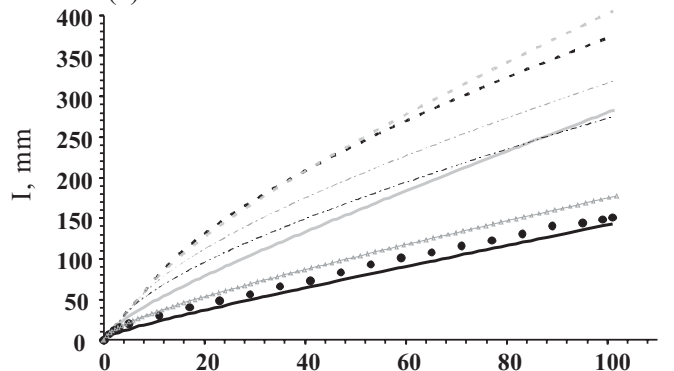

(f)

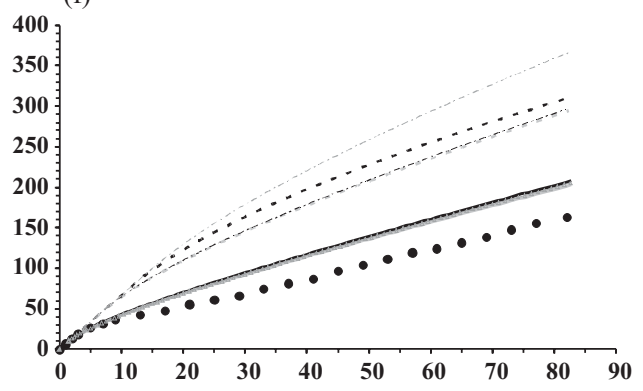

TEMPO, $\min$

Figura 1. Taxas de infiltração (a), (c) e (e) e lâminas infiltradas (b), (d) e (f) medidas e estimadas para os três testes experimentais realizados no LVA. 
(a)
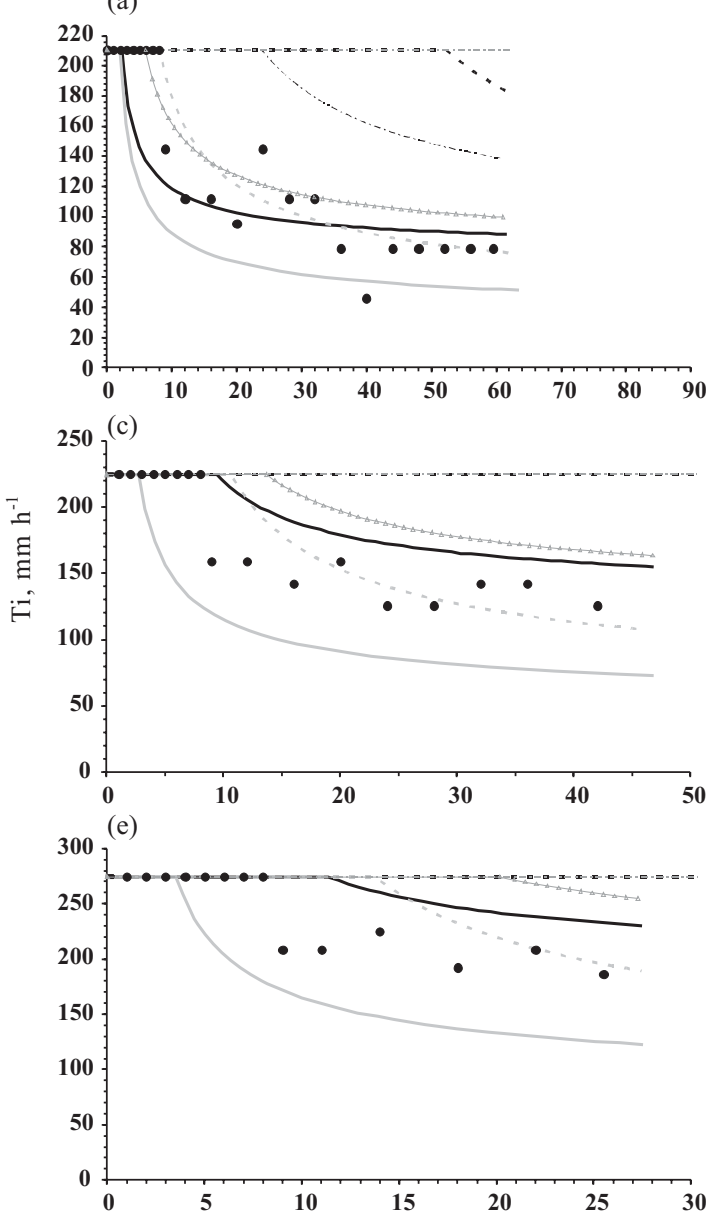

(b)
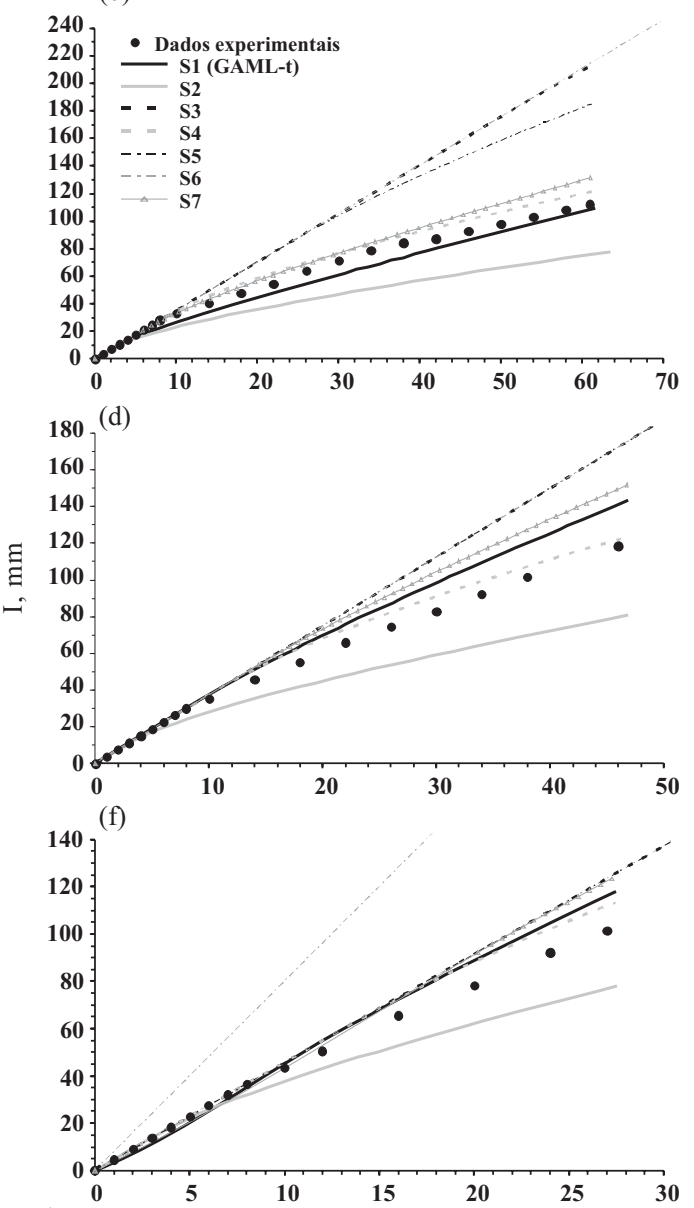

Figura 2. Taxas de infiltração (a), (c) e (e) e lâminas infiltradas (b), (d) e (f) medidas e estimadas para os três testes experimentais realizados no $\mathrm{PV}$.

teste experimental, que apresentou $\mathrm{K}_{0}$ bem maior que a Tie, causando superestimativa de Ti e I. A proximidade entre as simulações deveu-se tanto à semelhança entre os valores de $\mathrm{K}_{0}$ e Tie no primeiro e terceiro testes experimentais, como à proximidade entre os valores de $\theta_{\mathrm{s}}$ e $\theta_{\mathrm{w}}$, verificada pela relação $\theta_{\mathrm{s}} /$ $\theta_{\mathrm{w}}$ igual a 0,96 , em média. O pior desempenho de $\mathrm{S} 2$, comparativamente a $\mathrm{S} 1$ e $\mathrm{S} 7$, no segundo teste experimental permite questionar o uso do valor de $\mathrm{K}_{0}$, determinado com o método do permeâmetro de carga constante, para estimativa da infiltração, uma vez que as simulações S1 e S7 utilizam-se da Tie para estimar a condutividade hidráulica do perfil de solo. O melhor desempenho geral nos três testes experimentais pode ser atribuído às simulações $\mathrm{S} 1$ (GAML-t) e S7, esta última possui tendência de superestimar a infiltração, o que não foi observado na simulação feita com o GAML-t.

Com relação ao PV (Figura 2 e Quadro 5), observase que as simulações S3, S5 e S6 apresentaram sempre as piores estimativas de $\mathrm{Ti}$, decorrentes da grande falha de todas em estimar o tempo de empoçamento, simulando taxas de infiltração iguais a $i_{p}$ em praticamente todo o tempo de duração dos testes experimentais. Assim, suas curvas simuladas foram praticamente iguais durante o tempo de duração dos testes. Também se verifica a tendência da S2 (Mein \& Larson, 1973) em subestimar a infiltração em todos os testes, não induzindo a resultados confiáveis para Ti, conforme indicam os índices c'. A simulação S7, embora tenha mostrado boas estimativas de I, acabou deixando a desejar na estimativa de Ti, por apresentar superestimativa no tempo de empoçamento nos testes em que o valor da Tie foi maior (segundo e terceiro, com 132 e $196 \mathrm{~mm} \mathrm{~h}^{-1}$, respectivamente). De modo geral, as melhores estimativas de Ti e I foram obtidas por S1 (GAML-t) e S4.

As simulações S3, S4 e S6, realizadas de acordo com as adequações propostas por Cecílio et al. (2003), para o solo LV (Figura 3 e Quadro 5), não resultaram em estimativas viáveis tanto de Ti quanto de I; S4 e S6 apresentaram grande superestimativa do tempo de empoçamento, prevendo Ti igual a $i_{p}$ para quase todos os pontos experimentais. A simulação S7 mostrou 
(a)

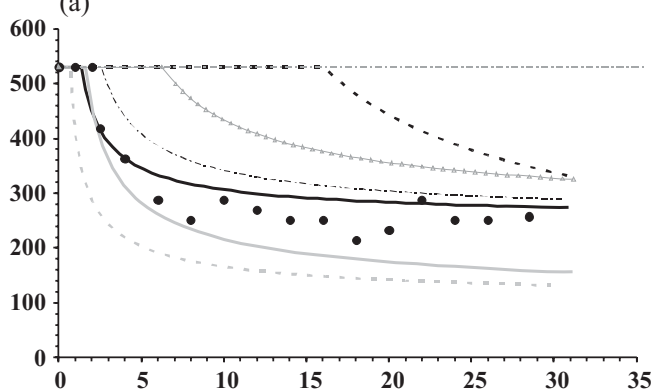

(c)

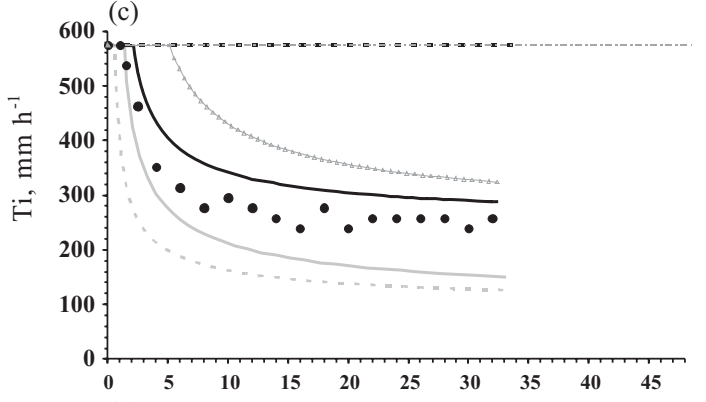

(e)

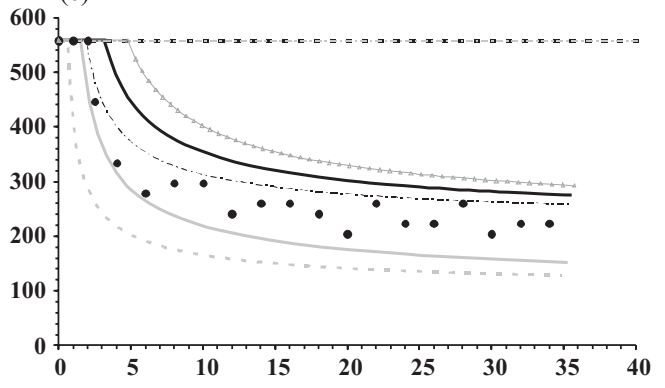

(b)
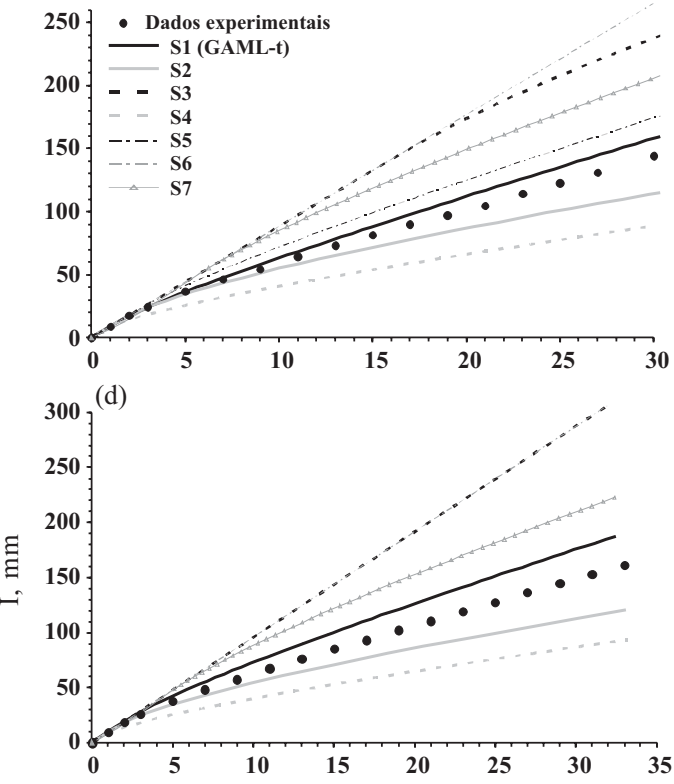

(f)

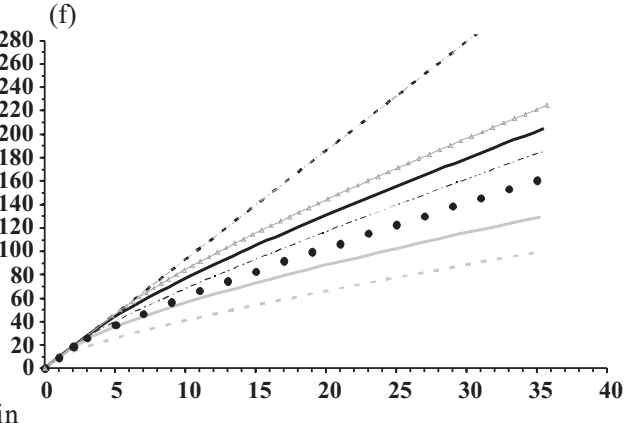

Figura 3. Taxas de infiltração (a), (c) e (e) e lâminas infiltradas (b), (d) e (f) medidas e estimadas para os três testes experimentais realizados no LV.

estimativas um pouco melhores, todavia ainda ruins, com tendência de superestimar os valores experimentais de Ti e I em todos os testes. As simulações S1 (GAML-t) e S5 apresentaram as melhores estimativas de Ti e I para os três testes experimentais, sendo a estimativa de S5 de melhor qualidade.

Uma análise geral dos experimentos e simulações realizadas para os três tipos de solo permite verificar que o GAML-t proveu boas estimativas da infiltração tanto para condições iniciais de baixa umidade do solo (testes do LV) quanto para condições iniciais de umidade mais elevada. Essa situação difere da apresentada por Chong et al. (1982), que recomendam a utilização do GAML apenas em condições iniciais de baixa umidade inicial do solo.

Observou-se que as sugestões para adequação dos parâmetros de entrada do GAML feitas por Cecílio et al. (2003), embora resultassem em estimativas promissoras para um tipo de solo, evidenciaram desempenho sofrível para outro, como é o caso das boas estimativas feitas por S5 no solo LV, que não se repetiram no LVA e no PV, e também da simulação S4 no solo PV, que mostrou desempenho fraco nos solos LV e LVA. Isso se deve ao fato de essas adequações se basearem em estimativas dos parâmetros de entrada feitas de modo empírico e, portanto, não aplicáveis a qualquer solo e qualquer situação. Assim, a utilização dessas estimativas empíricas pode acabar constituindo grave entrave à aplicação não apenas do GAML, mas de qualquer modelo com forte base física, conforme também relatado por Wilcox et al. (1990). O fraco desempenho, em muitos casos, de $\mathrm{S} 4$ e S5, que estimam $\psi_{\mathrm{f}}$ em função de características do solo, corrobora as afirmações de Yu (1999), segundo o qual a estimativa dos parâmetros de GA feita com a utilização de características do solo é usualmente inadequada.

As simulações que empregaram a Tie revelaramse, em geral, mais promissoras que aquelas que utilizaram $\mathrm{K}_{0}$ determinado com o uso do permeâmetro de carga constante. Como a Tie é um parâmetro cuja determinação é de maior confiabilidade que $\mathrm{K}_{0}$, recomenda-se sua utilização em estudos relativos à 
Quadro 5. Coeficientes estatísticos calculados para estimativas da lâmina infiltrada e da taxa de infiltração nos testes experimentais realizados

\begin{tabular}{|c|c|c|c|c|c|c|c|c|}
\hline \multirow{2}{*}{ Solo } & \multirow{2}{*}{\multicolumn{2}{|c|}{ Simulação }} & \multicolumn{2}{|c|}{ Primeiro teste } & \multicolumn{2}{|c|}{ Segundo teste } & \multicolumn{2}{|c|}{ Terceiro teste } \\
\hline & & & $c^{\prime}$ & Desempenho & c' & Desempenho & $\mathbf{c}^{\prime}$ & Desempenho \\
\hline \multirow[t]{14}{*}{ LVA } & $\mathrm{S} 1$ & I & 0,98 & Ótimo & 0,89 & Ótimo & 0,76 & Muito bom \\
\hline & & $\mathrm{Ti}$ & 0,72 & Bom & 0,64 & Mediano & 0,66 & Bom \\
\hline & $\mathrm{S} 2$ & I & 0,98 & Ótimo & 0,51 & Sofrível & 0,78 & Muito bom \\
\hline & & $\mathrm{Ti}$ & 0,79 & Muito bom & 0,21 & Péssimo & 0,66 & Bom \\
\hline & S3 & I & 0,63 & Mediano & 0,30 & Péssimo & 0,41 & Mau \\
\hline & & $\mathrm{Ti}$ & 0,59 & Sofrível & 0,06 & Péssimo & 0,13 & Péssimo \\
\hline & $\mathrm{S} 4$ & I & 0,61 & Mediano & 0,29 & Péssimo & 0,45 & Mau \\
\hline & & $\mathrm{Ti}$ & 0,52 & Sofrível & 0,01 & Péssimo & 0,15 & Péssimo \\
\hline & S5 & I & 0,55 & Sofrível & 0,46 & Mau & 0,45 & Mau \\
\hline & & $\mathrm{Ti}$ & 0,38 & Péssimo & 0,28 & Péssimo & 0,14 & Péssimo \\
\hline & $\mathrm{S} 6$ & I & 0,49 & Mau & 0,37 & Péssimo & 0,35 & Péssimo \\
\hline & & $\mathrm{Ti}$ & 0,22 & Péssimo & 0,15 & Péssimo & 0,01 & Péssimo \\
\hline & $\mathrm{S} 7$ & I & 0,88 & Ótimo & 0,86 & Ótimo & 0,77 & Muito bom \\
\hline & & $\mathrm{Ti}$ & 0,80 & Muito bom & 0,80 & Muito bom & 0,65 & Bom \\
\hline \multirow[t]{14}{*}{ PV } & $\mathrm{S} 1$ & I & 0,91 & Ótimo & 0,90 & Ótimo & 0,94 & Ótimo \\
\hline & & $\mathrm{Ti}$ & 0,58 & Sofrível & 0,66 & Bom & 0,46 & $\mathrm{Mau}$ \\
\hline & $\mathrm{S} 2$ & I & 0,67 & Bom & 0,75 & Muito bom & 0,83 & Muito bom \\
\hline & & $\mathrm{Ti}$ & 0,45 & Mau & 0,36 & Péssimo & 0,31 & Péssimo \\
\hline & S3 & I & 0,66 & Bom & 0,82 & Muito bom & 0,91 & Ótimo \\
\hline & & $\mathrm{Ti}$ & 0,01 & Péssimo & 0,03 & Péssimo & 0,11 & Péssimo \\
\hline & $\mathrm{S} 4$ & I & 0,95 & Ótimo & 0,96 & Ótimo & 0,95 & Ótimo \\
\hline & & $\mathrm{Ti}$ & 0,85 & Muito bom & 0,76 & Muito bom & 0,55 & Sofrível \\
\hline & S5 & I & 0,71 & Bom & 0,82 & Muito bom & 0,91 & Ótimo \\
\hline & & $\mathrm{Ti}$ & 0,31 & Péssimo & 0,03 & Péssimo & 0,11 & Péssimo \\
\hline & S6 & I & 0,66 & Bom & 0,82 & Muito bom & 0,91 & Ótimo \\
\hline & & $\mathrm{Ti}$ & 0,01 & Péssimo & 0,03 & Péssimo & 0,11 & Péssimo \\
\hline & $\mathrm{S} 7$ & I & 0,91 & Ótimo & 0,87 & Ótimo & 0,92 & Ótimo \\
\hline & & $\mathrm{Ti}$ & 0,78 & Muito bom & 0,53 & Sofrível & 0,23 & Péssimo \\
\hline \multirow[t]{14}{*}{ LV } & $\mathrm{S} 1$ & I & 0,92 & Ótimo & 0,84 & Muito bom & 0,76 & Muito bom \\
\hline & & $\mathrm{Ti}$ & 0,70 & Bom & 0,64 & Mediano & 0,50 & Sofrível \\
\hline & $\mathrm{S} 2$ & I & 0,82 & Muito bom & 0,74 & Bom & 0,80 & Muito bom \\
\hline & & $\mathrm{Ti}$ & 0,53 & Sofrível & 0,51 & Sofrível & 0,61 & Mediano \\
\hline & $\mathrm{S} 3$ & I & 0,58 & Sofrível & 0,50 & Mau & 0,48 & Mau \\
\hline & & $\mathrm{Ti}$ & 0,01 & Péssimo & 0,01 & Péssimo & 0,01 & Péssimo \\
\hline & $\mathrm{S} 4$ & I & 0,54 & Sofrível & 0,48 & Mau & 0,52 & Sofrível \\
\hline & & $\mathrm{Ti}$ & 0,37 & Péssimo & 0,34 & Péssimo & 0,39 & Péssimo \\
\hline & S5 & I & 0,81 & Muito bom & 0,83 & Muito bom & 0,87 & Ótimo \\
\hline & & $\mathrm{Ti}$ & 0,55 & Sofrível & 0,64 & Mediano & 0,75 & Bom \\
\hline & $\mathrm{S} 6$ & I & 0,54 & Sofrível & 0,50 & Mau & 0,48 & Mau \\
\hline & & $\mathrm{Ti}$ & 0,01 & Péssimo & 0,01 & Péssimo & 0,01 & Péssimo \\
\hline & $\mathrm{S} 7$ & I & 0,66 & Bom & 0,66 & Bom & 0,68 & Bom \\
\hline & & $\mathrm{Ti}$ & 0,26 & Péssimo & 0,31 & Péssimo & 0,39 & Péssimo \\
\hline
\end{tabular}


infiltração de água no solo. Contudo, é importante ressaltar que diversos estudos revelam que valores de Tie determinados por intermédio do método de anéis concêntricos são maiores que os determinados com o emprego de infiltrômetros de aspersão ou simuladores de chuva (Costa et al., 1999; Coelho et al., 2000; Pott \& De Maria, 2003). Esse fato é devido a dois fatores: (a) ao encrostamento da superfície causado pelo impacto direto das gotas de chuva sobre a superfície do solo, que ocorre na utilização dos simuladores de chuva e não ocorre no caso dos anéis concêntricos; e (b) à maior carga hidráulica sobre a superfície do solo ocasionada pelo uso dos anéis concêntricos. Assim, a Tie deve ser determinada usando-se simulador de chuvas para estimativa da infiltração em área submetida à irrigação por aspersão (Costa et al., 1999) ou sob precipitação. Considera-se que o uso de equações que estimem a redução da Tie em função da energia cinética de precipitação incidente sobre a superfície do solo, conforme realizado por Silva \& Kato (1998), pode fazer com que valores de Tie determinados pelo método dos anéis concêntricos sejam utilizados na estimativa de infiltração sob condições de precipitação. Todavia, é necessário que sejam realizadas pesquisas que comprovem tal hipótese.

Dentre as simulações, constatou-se que a única com viabilidade para todos os solos foi a S1 (GAML-t), o que se deve a dois fatores. $\mathrm{O}$ primeiro diz respeito à estimativa de $\psi_{\mathrm{f}}$ proposta por Slack (1980), que considera a variação de umidade ao longo de toda a frente de umedecimento entre os valores de $\theta_{\mathrm{i}}$ e $\theta_{\mathrm{w}}$. O segundo fator deve-se à utilização da Tie em substituição à condutividade hidráulica dos solos em todo o perfil, por ser este um parâmetro mais confiável, uma vez que seu método de determinação não causa alteração na estrutura do solo. Dessa forma, recomenda-se a utilização da adequação do GAML proposta neste trabalho para estimativa da infiltração de água no solo, em substituição às demais opções.

\section{CONCLUSÕES}

1. A substituição da Tie pela condutividade hidráulica do solo saturado produziu estimativas mais coerentes da infiltração da água do solo.

2. As substituições dos parâmetros de entrada do GAML baseadas em equações empíricas não se apresentaram confiáveis para estimativa da infiltração.

3. A adequação dos parâmetros de entrada do modelo de GAML proposta (GAML-t) foi capaz de prover estimativas promissoras da infiltração de água em todos os solos estudados.

4. Das adequações do modelo de GAML avaliadas, o GAML-t apresentou melhores resultados, analisandose conjuntamente os três tipos de solo.

\section{LITERATURA CITADA}

ALEXANDER, L. \& SKAGGS, R.W. Predicting unsaturated hydraulic conductivity from the soil water characteristic. Trans. Am. Soc. Agric. Eng., 29:176-184, 1986.

BRAKENSIEK, D.L. \& ONSTAD, C.A. Parameter estimation of the Green and Ampt infiltration equation. Water Res. Res., 13:1009-1012, 1977.

BRANDÃO, V.S.; CECÍLIO, R.A.; PRUSKI, F.F. \& SILVA, D.D. Infiltração da água no solo. Viçosa, MG, Universidade Federal de Viçosa, 2006. 120p.

BROOKS, R.H. \& COREY, A.T. Hydraulic properties of porous media. Fort Collins, Colorado State University, 1964. 26p. (Hydrologic Paper, 3)

CAMARGO, A.P. \& SENTELHAS, P.C. Avaliação do desempenho de diferentes métodos de estimativa da evapotranspiração potencial no Estado de São Paulo, Brasil. R. Bras. Agrometeorol., 5:89-97, 1997.

CECÍLIO, R.A.; SILVA, D.D.; PRUSKI, F.F. \& MARTINEZ, M.A. Modelagem da infiltração de água no solo sob condições de estratificação utilizando-se a equação de Green-Ampt. R. Bras. Eng. Agric. Amb., 7:415-422, 2003.

CHONG, S.K.; GREEN, R.E. \& AHUJA, L.R. Infiltration prediction based on estimation of Green-Ampt wetting front pressure head from measurements of soil water redistribution. Soil Sci. Soc. Am. J., 46:235-239, 1982.

COELHO, R.D.; MIRANDA, J.H. \& DUARTE, S.N. Infiltração de água no solo: Parte I. Infiltrômetro de anéis versus infiltrômetro de aspersores. R. Bras. Eng. Agric. Amb., 4:137-141, 2000.

COSTA, É.L.; SILVA, A.M.; COLOMBO, A. \& ABREU, A.R. Infiltração de água em solo, determinada por simulador de chuvas e pelo método dos anéis. R. Bras. Eng. Agric. Amb., 3:131-134, 1999.

LEGATES, D.R. \& McCABE Jr., G.J. Evaluating the use of "goodness-of-fit" measures in hydrologic and hydroclimatic model validation. Water Res. Res., 35:233-241, 1999.

MEIN, R.G. \& LARSON, C.L. Modeling infiltration during a steady rain. Water Res. Res., 9:384-94, 1973

MELLO, L.T.A. Avaliação de metodologias para obtenção dos parâmetros do modelo de Green-Ampt modificado por Mein e Larson. Viçosa, MG, Universidade Federal de Viçosa, 2003. 77p. (Tese de Mestrado)

MESQUITA, M.G.B.F. \& MORAES, S.O. A dependência entre a condutividade hidráulica saturada e atributos físicos do solo. Ci. Rural, 34:963-969, 2004.

PALACIOS-VÉLEZ, O.L.; GANDOY-BERNASCONI, W. \& CUEVAS-RENAUD, B. Geometric analysis of surface runoff and the computation order of unit elements in distributed hydrological models. J. Hydrol., 211:266-274, 1998.

POTT, C.A. \& DE MARIA, I.C. Comparação de métodos de campo para determinação da velocidade de infiltração básica. R. Bras. Ci. Solo, 27:19-27, 2003.

SILVA, C.L. \& KATO, E. Avaliação de modelos para previsão da infiltração de água em solos sob cerrado. Pesq. Agropec. Bras., 33:1149-1158, 1998. 
SLACK, D.C. Modeling infiltration under moving sprinkler irrigation systems. Trans. Am. Soc. Agric. Eng., 23:596600, 1980.

WILCOX, B.P.; RAWLS, W.J.; BRAKENSIEK, D.L. \& WIGHT, J.R. Predicting runoff from rangeland catchments: A comparison of two models. Water Res. Res., 26:2401-2410, 1990.
WILLMOTT, C.J. On the validation of models. Phys. Geog., 2:184-194, 1981.

YU, B. A comparison of the Green-Ampt and a spatially variable infiltration model for natural storm events. Trans. Am. Soc. Agric. Eng., 42:88-97, 1999. 